\title{
CULTURA DO LIMÃO NO BRASIL: CUSTO DE PRODUÇÃO E LUCRATIVIDADE
}

Flavio Alberto Oliva, Maycon Vieira Amin, Douglas Fernandes, Ana Paula Verginassi Pocaia, Bruna Coelho de Lima, Layane Oliveira Carvalho, Marcus Ayrton Rocha de Lima

Universidade do Oeste Paulista - UNOESTE, Faculdade de Ciências Agrárias, Presidente Prudente, SP. E-mail: flavioaoliva@gmail.com

\section{RESUMO}

Na Grécia Antiga, o limão tinha importância pelas suas propriedades aromatizantes e como objeto de decoração. No Egito Antigo era utilizado no processo de mumificação. Atualmente o limão é utilizado pela maioria dos países como ingrediente da gastronomia e para fins medicinais. 0 objetivo do trabalho foi apresentar a mensuração de custos e resultados da produção do limão, subsidiando indicadores necessários para tomada de decisão gerencial e estratégica em propriedades rurais. A metodologia propôs-se a contribuir na organização das informações obtidas em planilhas adequadas possibilitando assim a apuração confiável dos custos e das receitas e, consequentemente, proporcionando informações fidedignas para a estruturação de informações necessárias à gestão da cultura do limão. O estudo baseou-se em informações e dados quantitativos dos anos de 2016 e 2017. Como resultado, evidenciou-se a viabilidade econômicofinanceira da cultura.

Palavras-chave: Citricultura, Medicinal, Custo, Resultado, Financeiro.

\section{LEMON CULTURE IN BRAZIL: COST OF PRODUCTION AND PROFITABILITY}

\begin{abstract}
In Ancient Greece, lemon was important for its flavoring properties and as an object of decoration. In Ancient Egypt it was used in the process of mummification. Lemon is currently used by most countries as an ingredient in gastronomy and for medicinal purposes. The objective of this work was to present the measurement of costs and results of lemon production, subsidizing indicators necessary for managerial and strategic decision making in rural properties. The methodology proposed to contribute to the organization of the information obtained in appropriate spreadsheets, thus enabling reliable calculation of costs and revenues and, consequently, providing reliable information for the structuring of information necessary for the management of garlic culture. The study was based on information and quantitative data from the years 2016 and 2017. As a result, the economic-financial viability of the crop was evidenced.
\end{abstract}

Keywords: Citrus, Medicinal, Cost, Outcome, Financial

\section{INTRODUÇÃO}

A citricultura é uma significativa atividade do agronegócio brasileiro. As frutas que estão neste grupo são do gênero Citrus, visto que as principais espécies são: as laranjas doces, as tangerinas, os limões, as limas ácidas, os pomelos, e demais espécies classificadas como menos conhecidas (DONADIO; STUCHI; CYRILLO, 1998).

As primeiras plantas cítricas foram ingressas no Brasil através dos portugueses, trazendo as mudas da Espanha, logo no inicio da colonização (NEVES; JANK, 2006). O intuito em 
trazer essas plantas, era de propor um abastecimento de vitamina $\mathrm{C}$ para ser usada como antídoto do escorbuto, doença que exterminava grande parte das tripulações na época (NEVES; JANK, 2006).

Essas mudas se adaptaram bem ao clima, possibilitando que se espalhassem por todo o território brasileiro. Porém, desde o começo, a região Centro-Sul obteve maior destaque de produção, devido às condições edafoclimáticas e proximidade com o mercado consumidor. Desta forma, São Paulo é visto até os dias de hoje pelo domínio desse setor, sendo que das 1.178 máquinas extratoras de suco de laranja instaladas no país, 1.061 encontram-se no estado de São Paulo, 72 no sul e 45 no Nordeste (NEVES et al., 2010).

A lima-ácida "Tahiti" (Citrus latifolia Tanaka), famosa entre os consumidores brasileiros como limão Tahiti, é tida como uma das preciosidades da citricultura. É uma variedade de citro americana, oriunda da California, Estados Unidos, onde brotou derivada de sementes de limão trazidas do Tahiti, por volta de 1870 . Seu cultivo se propagou pelas três Américas, o único continente onde este, é produzido comercialmente. A produção de limão Tahiti vem aumentando e se tornando cada vez mais importante na citricultura brasileira, com elevada exportação além da demanda no mercado interno. (BARROS, S. D. et al., 1991).

Segundo a revista Dinheiro Rural (2016), tendo como fonte a Organização das Nações Unidas para a Alimentação e a Agricultura (FAO), os cinco maiores produtores mundiais de limão são, Índia, México, China, Argentina e Brasil.

Em 2012 o Brasil finalizou com uma produção total de 1,2 milhão de toneladas de limão, sendo os principais estados produtores, São Paulo com a produção de 923.178 ton., Minas Gerais 88.329 ton., Bahia 55.433 ton., Pará com 23.112, Rio de Janeiro 18.554 ton. e outros estados estimando 99.669 toneladas. A área total colhida foi de 47 mil hectares, tendo como destaque, São Paulo com 76\% do rendimento nacional (EMBRAPA, 2012).

\section{METODOLOGIA}

O estudo propôs-se a contribuir na organização das informações obtidas em planilhas adequadas possibilitando assim a apuração confiável dos custos e das receitas e, consequentemente, proporcionando informações fidedignas para a estruturação de informações necessárias à gestão da cultura do limão. O estudo baseou-se em informações e dados quantitativos dos anos de 2016 e 2017, no entanto, possui também caráter qualitativo á medida que se utilizou dessas informações para análises financeiras, econômicas e tomadas de decisões.

\section{RESULTADOS}

Por ser uma cultura permanente, o custo com insumos foi projetado para seis anos, sendo que, no primeiro ano ocorre o maior desembolso financeiro devido a utilização das mudas, ou seja, $40,59 \%$ do custo no período em questão, estão concentrados no primeiro ano da atividade. A partir do segundo ano, conforme Tabela 1, os dispêndios financeiros com insumos estão voltados à manutenção da plantação. 
Tabela 1. Custo dos Insumos

\begin{tabular}{|c|c|c|c|c|c|c|c|c|c|c|c|c|c|c|}
\hline & \multirow{3}{*}{$\begin{array}{l}U \\
\text { ni }\end{array}$} & \multirow{3}{*}{$\begin{array}{l}\text { Custo } \\
\text { Unitár } \\
\text { io }\end{array}$} & \multicolumn{12}{|c|}{ Período } \\
\hline & & & \multicolumn{2}{|c|}{10 ano } & \multicolumn{2}{|c|}{$2 \stackrel{0}{ }$ ano } & \multicolumn{2}{|c|}{ 3o ano } & \multicolumn{2}{|c|}{$4 \%$ ano } & \multicolumn{2}{|c|}{ 5o ano } & \multicolumn{2}{|c|}{60 ano } \\
\hline & & & $\begin{array}{l}\text { Qt } \\
d\end{array}$ & $\begin{array}{c}\text { Custo } \\
\mathrm{R} \$\end{array}$ & $\begin{array}{l}\text { Qt } \\
d\end{array}$ & $\begin{array}{c}\text { Custo } \\
\mathrm{R} \$\end{array}$ & $\begin{array}{l}\text { Qt } \\
d\end{array}$ & $\begin{array}{c}\text { Custo } \\
\mathrm{R} \$\end{array}$ & $\begin{array}{l}\text { Qt } \\
d\end{array}$ & $\begin{array}{c}\text { Custo } \\
\mathrm{R} \$\end{array}$ & $\begin{array}{l}\text { Qt } \\
d\end{array}$ & $\begin{array}{c}\text { Custo } \\
\mathrm{R} \$\end{array}$ & $\begin{array}{l}\text { Qt } \\
d\end{array}$ & $\begin{array}{c}\text { Custo } \\
\mathrm{R} \$\end{array}$ \\
\hline 1. INSUMOS & & & & & & & & & & & & & & \\
\hline $\begin{array}{l}\text { Mudas (Plantio e } \\
\text { Replantio) }\end{array}$ & ud & 7,75 & $\begin{array}{c}36 \\
6\end{array}$ & 2836,5 & & & & & & & & & & \\
\hline Calcário & $\mathrm{t}$ & $\begin{array}{c}162,5 \\
0\end{array}$ & $\begin{array}{c}1,0 \\
6 \\
\end{array}$ & 172,25 & & & 1 & 162,50 & & & 1 & 162,50 & & \\
\hline NPK & $\mathrm{Kg}$ & 4,03 & 20 & 80,60 & 30 & 120,90 & 45 & 181,35 & 65 & 261,95 & 80 & 322,40 & 90 & 362,70 \\
\hline Sulfato de magnésio & $\mathrm{Kg}$ & 2,85 & 0,4 & 1,14 & $\begin{array}{l}0 \\
6\end{array}$ & 1,71 & $\begin{array}{l}1, \\
3\end{array}$ & 3,71 & 2 & 5,70 & $\begin{array}{l}2 \\
7\end{array}$ & 7,70 & 4 & 11,40 \\
\hline Sulfato de zinco & $\mathrm{Kg}$ & 3,12 & 0,4 & 1,25 & $\begin{array}{l}0 \\
6\end{array}$ & 1,87 & $\begin{array}{l}1, \\
3\end{array}$ & 4,06 & 2 & 6,24 & $\begin{array}{l}2, \\
7\end{array}$ & 8,42 & 5 & 15,60 \\
\hline Sulfato de manganês & $\mathrm{Kg}$ & 7,81 & 0,4 & 3,12 & $\begin{array}{l}0, \\
6\end{array}$ & 4,69 & $\begin{array}{l}1, \\
3\end{array}$ & 10,15 & 2 & 15,62 & $\begin{array}{l}2, \\
7\end{array}$ & 21,09 & 5 & 39,05 \\
\hline Sulfato de cobre & $\mathrm{Kg}$ & 8,93 & & & & & 2 & 17,86 & $\begin{array}{l}2, \\
5\end{array}$ & 22,33 & $\begin{array}{l}2 \\
5\end{array}$ & 22,33 & 4 & 35,72 \\
\hline Adubo orgânico & $\mathrm{t}$ & $\begin{array}{c}110,0 \\
0\end{array}$ & 2 & 220,00 & & & 2 & 220,00 & & & 2 & 220,00 & & \\
\hline Formicida & $\mathrm{Kg}$ & 8,00 & 4 & 32,00 & 4 & 32,00 & 3 & 24,00 & 3 & 24,00 & 2 & 16,00 & 2 & 16,00 \\
\hline Herbicida & L & 21,85 & & & & & 2 & 43,70 & 2 & 43,70 & 2 & 43,70 & 2 & 43,70 \\
\hline Inseticida & L & 38,85 & 1 & 38,85 & 1 & 38,85 & $\begin{array}{l}3, \\
6\end{array}$ & 139,86 & $\begin{array}{l}3 \\
6\end{array}$ & 139,86 & $\begin{array}{l}5, \\
8\end{array}$ & 225,33 & 7 & 271,95 \\
\hline Fungicida & $\mathrm{Kg}$ & 20,62 & 1 & 20,62 & 2 & 41,24 & 3 & 61,86 & 3 & 61,86 & 6 & 123,72 & 6 & 123,72 \\
\hline Espalhante adesivo & $\mathrm{L}$ & 6,90 & 1 & 6,90 & 2 & 13,80 & 2 & 13,80 & 2 & 13,80 & 4 & 27,60 & 6 & 41,40 \\
\hline Outros insumos & $\mathrm{Kg}$ & 2,00 & & & $\begin{array}{c}11 \\
0 \\
\end{array}$ & 220,00 & $\begin{array}{c}11 \\
0 \\
\end{array}$ & 220,00 & $\begin{array}{c}11 \\
0\end{array}$ & 220,00 & $\begin{array}{c}11 \\
0\end{array}$ & 220,00 & $\begin{array}{c}11 \\
0\end{array}$ & 220,00 \\
\hline SUB TOTAL INSUMOS & $\mathrm{R} \$$ & & & $\begin{array}{c}3.413 \\
23\end{array}$ & & 475,06 & & $\begin{array}{c}1.102 \\
84\end{array}$ & & 815,06 & & $\begin{array}{c}1.420 \\
78\end{array}$ & & $\begin{array}{c}1.181 \\
24\end{array}$ \\
\hline
\end{tabular}

Em relação aos serviços ( Tabela 2), o custo no período de seis anos foi calculado em $\mathrm{R} \$ 20.580,00$ sendo $19,58 \%$ do montante no primeiro ano, $12,15 \%$ no segundo ano, $14,58 \%$ no terceiro ano, $15,06 \%$ no quarto ano, $18,71 \%$ no quinto ano e $19,92 \%$ no sexto ano de atividade. 
Tabela 2. Custo dos Serviços

\begin{tabular}{|c|c|c|c|c|c|c|c|c|c|c|c|c|c|c|}
\hline & \multirow{3}{*}{$\begin{array}{c}\text { Un } \\
i\end{array}$} & \multirow{3}{*}{$\begin{array}{c}\text { Custo } \\
\text { Unitár } \\
\text { io }\end{array}$} & \multicolumn{12}{|c|}{ Período } \\
\hline & & & \multicolumn{2}{|r|}{ 10 ano } & \multicolumn{2}{|c|}{20 ano } & \multicolumn{2}{|c|}{ 3o ano } & \multicolumn{2}{|c|}{ 4ㅇ ano } & \multicolumn{2}{|c|}{ 5o ano } & \multicolumn{2}{|c|}{ 6o ano } \\
\hline & & & $\begin{array}{c}\text { Qt } \\
d\end{array}$ & Custo & $\begin{array}{l}\text { Qt } \\
d\end{array}$ & Custo & $\begin{array}{c}\text { Qt } \\
d\end{array}$ & Custo & $\begin{array}{c}\text { Qt } \\
d\end{array}$ & Custo & $\begin{array}{c}\text { Qt } \\
d\end{array}$ & Custo & $\begin{array}{l}\text { Qt } \\
d\end{array}$ & Custo \\
\hline \multicolumn{15}{|l|}{ 2. SERVIÇOS } \\
\hline Preparacao do solo & $\begin{array}{l}\mathrm{h} / \\
\mathrm{M}\end{array}$ & 50,00 & 10 & $\begin{array}{c}500,0 \\
0\end{array}$ & & & & & & & & & & \\
\hline Aplicação de Calcário & $\begin{array}{l}\mathrm{d} / \\
\mathrm{H}\end{array}$ & 50,00 & 2 & $\begin{array}{c}100,0 \\
0\end{array}$ & & & & & & & & & & \\
\hline $\begin{array}{l}\text { Marcação de curvas de } \\
\text { nível }\end{array}$ & $\begin{array}{l}\mathrm{d} / \\
\mathrm{H}\end{array}$ & 50,00 & 1 & 50,00 & & & & & & & & & & \\
\hline $\begin{array}{l}\text { Marcação de covas e } \\
\text { coveamento }\end{array}$ & $\begin{array}{l}\mathrm{d} / \\
\mathrm{H}\end{array}$ & 50,00 & 10 & $\begin{array}{c}500,0 \\
0\end{array}$ & & & & & & & & & & \\
\hline $\begin{array}{l}\text { Preparação e adubação da } \\
\text { cova }\end{array}$ & $\begin{array}{l}\mathrm{d} / \\
\mathrm{H}\end{array}$ & 50,00 & 3 & $\begin{array}{c}150,0 \\
0\end{array}$ & & & & & & & & & & \\
\hline $\begin{array}{l}\text { Construção de } \\
\text { carreadores }\end{array}$ & $\begin{array}{l}\mathrm{h} / \\
\mathrm{M}\end{array}$ & $\begin{array}{c}120,0 \\
0\end{array}$ & 4 & $\begin{array}{c}480,0 \\
0\end{array}$ & & & & & & & & & & \\
\hline Plantio e replantio & $\begin{array}{l}\mathrm{d} / \\
\mathrm{H}\end{array}$ & 50,00 & 3 & $\begin{array}{c}150,0 \\
0\end{array}$ & & & & & & & & & & \\
\hline $\begin{array}{l}\text { Adubação de cobertura } \\
(3 x)\end{array}$ & $\begin{array}{l}d / \\
H\end{array}$ & 50,00 & 4 & $\begin{array}{c}200,0 \\
0\end{array}$ & 4 & $\begin{array}{c}200,0 \\
0\end{array}$ & 5 & $\begin{array}{c}250,0 \\
0 \\
\end{array}$ & 5 & $\begin{array}{c}250,0 \\
0 \\
\end{array}$ & 6 & $\begin{array}{c}300,0 \\
0 \\
\end{array}$ & 6 & $\begin{array}{c}300,0 \\
0\end{array}$ \\
\hline Adubação foliar & $\begin{array}{l}\mathrm{d} / \\
\mathrm{H}\end{array}$ & 50,00 & & & & & & & & & 4 & $\begin{array}{c}200,0 \\
0\end{array}$ & 4 & $\begin{array}{c}200,0 \\
0\end{array}$ \\
\hline Desbaste de frutos & $\begin{array}{l}\mathrm{d} / \\
\mathrm{H}\end{array}$ & 50,00 & 1 & 50,00 & 2 & $\begin{array}{c}100,0 \\
0\end{array}$ & & & & & & & & \\
\hline Combate a formigas & $\begin{array}{l}\mathrm{d} / \\
\mathrm{H}\end{array}$ & $\begin{array}{c}100,0 \\
0\end{array}$ & 5 & $\begin{array}{c}500,0 \\
0\end{array}$ & 5 & $\begin{array}{c}500,0 \\
0\end{array}$ & 5 & $\begin{array}{c}500,0 \\
0\end{array}$ & 5 & $\begin{array}{c}500,0 \\
0\end{array}$ & 5 & $\begin{array}{c}500,0 \\
0\end{array}$ & 5 & $\begin{array}{c}500,0 \\
0\end{array}$ \\
\hline Aplicação de herbicidas & $\begin{array}{l}d / \\
H\end{array}$ & $\begin{array}{c}100,0 \\
0\end{array}$ & 1 & $\begin{array}{c}100,0 \\
0\end{array}$ & 2 & $\begin{array}{c}200,0 \\
0\end{array}$ & 2 & $\begin{array}{c}200,0 \\
0\end{array}$ & 2 & $\begin{array}{c}200,0 \\
0\end{array}$ & 3 & $\begin{array}{c}300,0 \\
0\end{array}$ & 3 & $\begin{array}{c}300,0 \\
0\end{array}$ \\
\hline Tratamento fitossanitário & $\begin{array}{l}\mathrm{d} / \\
\mathrm{H}\end{array}$ & $\begin{array}{c}100,0 \\
0\end{array}$ & 5 & $\begin{array}{c}500,0 \\
0\end{array}$ & 5 & $\begin{array}{c}500,0 \\
0\end{array}$ & 6 & $\begin{array}{c}600,0 \\
0\end{array}$ & 6 & $\begin{array}{c}600,0 \\
0\end{array}$ & 6 & $\begin{array}{c}600,0 \\
0\end{array}$ & 6 & $\begin{array}{c}600,0 \\
0\end{array}$ \\
\hline Capina + coroamento & $\begin{array}{l}\text { d/ } \\
H\end{array}$ & 50,00 & 13 & $\begin{array}{c}650,0 \\
0 \\
\end{array}$ & 13 & $\begin{array}{c}650,0 \\
0\end{array}$ & 13 & $\begin{array}{c}650,0 \\
0\end{array}$ & 10 & $\begin{array}{c}500,0 \\
0\end{array}$ & 10 & $\begin{array}{c}500,0 \\
0\end{array}$ & 10 & $\begin{array}{c}500,0 \\
0\end{array}$ \\
\hline Desbrota & $\begin{array}{l}\mathrm{d} / \\
\mathrm{H}\end{array}$ & 50,00 & 2 & $\begin{array}{c}100,0 \\
0\end{array}$ & 2 & $\begin{array}{c}100,0 \\
0\end{array}$ & 3 & $\begin{array}{c}150,0 \\
0\end{array}$ & 3 & $\begin{array}{c}150,0 \\
0\end{array}$ & 2 & $\begin{array}{c}100,0 \\
0\end{array}$ & 2 & $\begin{array}{c}100,0 \\
0 \\
\end{array}$ \\
\hline Colheita & $\begin{array}{l}\text { d/ } \\
H\end{array}$ & 50,00 & & & 2 & $\begin{array}{c}100,0 \\
0\end{array}$ & 6 & $\begin{array}{c}300,0 \\
0\end{array}$ & 9 & $\begin{array}{c}450,0 \\
0\end{array}$ & 13 & $\begin{array}{c}650,0 \\
0\end{array}$ & 16 & $\begin{array}{c}800,0 \\
0\end{array}$ \\
\hline Transporte colheita & $\begin{array}{l}\mathrm{d} / \\
\mathrm{H}\end{array}$ & 50,00 & & & 1 & 50,00 & 3 & $\begin{array}{c}150,0 \\
0\end{array}$ & 4 & $\begin{array}{c}200,0 \\
0\end{array}$ & 6 & $\begin{array}{c}300,0 \\
0\end{array}$ & 7 & $\begin{array}{c}350,0 \\
0\end{array}$ \\
\hline Transporte colheita & $\begin{array}{l}\mathrm{h} / \\
\mathrm{M}\end{array}$ & 50,00 & & & 1 & 50,00 & 1 & 50,00 & 1 & 50,00 & 2 & $\begin{array}{c}100,0 \\
0\end{array}$ & 2 & $\begin{array}{c}100,0 \\
0\end{array}$ \\
\hline Classificação e embalagem & $\begin{array}{l}\text { d/ } \\
H\end{array}$ & 50,00 & & & 1 & 50,00 & 3 & $\begin{array}{c}150,0 \\
0\end{array}$ & 4 & $\begin{array}{c}200,0 \\
0\end{array}$ & 6 & $\begin{array}{c}300,0 \\
0\end{array}$ & 7 & $\begin{array}{c}350,0 \\
0\end{array}$ \\
\hline SUB TOTAL SERVIÇOS & $\mathrm{R} \$$ & & & $\begin{array}{c}4.030 \\
00\end{array}$ & & $\begin{array}{c}2.500, \\
00\end{array}$ & & $\begin{array}{c}3.000, \\
00\end{array}$ & & $\begin{array}{c}3.100, \\
00\end{array}$ & & $\begin{array}{c}3.850 \\
00\end{array}$ & & $\begin{array}{c}4.100, \\
00\end{array}$ \\
\hline TOTAL & $\mathrm{R} \$$ & & & $\begin{array}{c}7.443, \\
23\end{array}$ & & $\begin{array}{c}2.975, \\
06\end{array}$ & & $\begin{array}{c}4.102, \\
84\end{array}$ & & $\begin{array}{c}3.915, \\
06\end{array}$ & & $\begin{array}{c}5.270 \\
78\end{array}$ & & $\begin{array}{c}5.281, \\
24\end{array}$ \\
\hline
\end{tabular}

Na implantação e manutenção de uma lavoura de limão, os custos com serviços são mais expressivos que os insumos (Tabela 3). Deve o produtor estar atento com os custos de mão de obra, avaliar a contratação temporária ou permanente do trabalhador. Deve ainda avaliar a aquisição de máquinas e equipamentos ou optar pelo aluguel. 
Tabela 3. Custo Total de Produção

\begin{tabular}{|c|c|c|c|c|c|c|}
\hline Custo Total de Produção & 1ㅇ Ano & 2ㅇ Ano & 3o Ano & 4ㅇ Ano & 5o Ano & 6o Ano \\
\hline Insumos & $3.413,23$ & 475,06 & $1.102,84$ & 815,06 & $1.420,78$ & $1.181,24$ \\
\hline Serviços & $4.030,00$ & $2.500,00$ & $3.000,00$ & $3.100,00$ & $3.850,00$ & $4.100,00$ \\
\hline Total & $7.443,23$ & $2.975,06$ & $4.102,84$ & $3.915,06$ & $5.270,78$ & $5.281,24$ \\
\hline
\end{tabular}

Não menos importante que o controle de custos, a comercialização do produto deve ser feita de maneira estratégica para garantir o melhor resultado sobre os investimentos e riscos imputados na citricultura.

Tabela 4. Preço, Custo de Produção e Resultado

\begin{tabular}{|c|c|c|c|c|c|c|c|}
\hline Mês & $\begin{array}{c}\text { Preço } \\
\text { Kg } \\
\text { R\$ }\end{array}$ & $\begin{array}{c}\text { Cust } \\
\text { Kg R\$ }\end{array}$ & $\begin{array}{c}\text { Resultad } \\
\text { o } \\
\text { Kg R\$ }\end{array}$ & $\begin{array}{c}\text { Produção Mensal } \\
\text { Kg }\end{array}$ & $\begin{array}{c}\text { Faturamento } \\
\text { R\$ }\end{array}$ & $\begin{array}{c}\text { Custo } \\
\text { R\$ }\end{array}$ & $\begin{array}{c}\text { Resultado } \\
\text { R\$ }\end{array}$ \\
\hline jun/16 & 1,40 & 0,60 & 0,80 & 1000 & $1.400,00$ & 600,00 & 800,00 \\
\hline jul/16 & 1,37 & 0,60 & 0,77 & 1000 & $1.370,00$ & 600,00 & 770,00 \\
\hline ago/16 & 1,80 & 0,60 & 1,20 & 1000 & $1.800,00$ & 600,00 & $1.200,00$ \\
\hline set/16 & 2,44 & 0,60 & 1,84 & 1000 & $2.440,00$ & 600,00 & $1.840,00$ \\
\hline out/16 & 2,74 & 0,60 & 2,14 & 1000 & $2.740,00$ & 600,00 & $2.140,00$ \\
\hline nov/16 & 2,50 & 0,60 & 1,90 & 1000 & $2.500,00$ & 600,00 & $1.900,00$ \\
\hline dez/16 & 1,13 & 0,60 & 0,53 & 1000 & $1.130,00$ & 600,00 & 530,00 \\
\hline jan/17 & 0,98 & 0,60 & 0,38 & 1000 & 980,00 & 600,00 & 380,00 \\
\hline fev/17 & 0,58 & 0,60 & $-0,02$ & 1000 & 580,00 & 600,00 & $-20,00$ \\
\hline mar/1 & & & & & & & \\
7 & 0,54 & 0,60 & $-0,06$ & 1000 & 540,00 & 600,00 & $-60,00$ \\
\hline abr/17 & 0,55 & 0,60 & $-0,05$ & 1000 & 550,00 & 600,00 & $-50,00$ \\
\hline mai/17 & 0,44 & 0,60 & $-0,16$ & 1000 & 440,00 & 600,00 & $-160,00$ \\
\hline Total & 16,47 & 7,20 & 9,27 & 12000 & $16.470,00$ & 0 & $9.270,00$ \\
\hline
\end{tabular}

\section{DISCUSSÃO}

A cultura do limão, como qualquer outra atividade da economia, requer o desenvolvimento de estratégias de produção, manejo e comercialização. Na Tabela 4, observamos nitidamente a variação de preços do produto e, como consequência o resultado. Os preços de mercado comportaram-se de maneira diferente entre o segundo semestre de 2016 e o primeiro semestre de 2017. Deve o produtor considerar as expressivas variações de preços e tendências de mercado para a melhor tomada de decisão.

É fundamental conhecer as variações sazonais bem como os movimentos de oferta e demanda para agir estrategicamente em busca do melhor retorno.

\section{CONCLUSÃO}

Considerando que há uma particularidade na variação do preço de mercado do limão, deve a cultura ser analisada ao longo do ano (Tabela 4) de maneira que possamos entender a sazonalidade e os efeitos da oferta e demanda. Embora apresentando severas variações entre um semestre e outro, a cultura é viável. A lucratividade foi calculada em $56,28 \%$, demonstrando que os meses de preços abaixo do custo de produção não inviabilizam a atividade. 


\section{REFERÊNCIAS}

BARROS, S. A. et al. Efeito do ácido giberélico e do uniconazole na fisiologia pós-colheita do limão Tahiti'(Citrus latifolia Tanaka). Revista Brasileira de Fruticultura, Cruz das Almas, v. 13, n. 3, p. 223-226, 1991.

DONADIO, L. C.; STUCHI, E. S.; CYRILLO, F. L. de L. Tangerinas ou mandarinas. Boletim Citrícola, Jaboticabal: Funep, n. 5, p. 01- 40, 1998.

DINHEIRO RURAL. 0 doce negocio do limão. 2016. Disponível em: https://www.dinheirorural.com.br/secao/agronegocios/o-doce-negocio-do-limao Acesso em: 8 jun. 2017.

EMBRAPA FRUTICULTURA. Produção brasileira de limão. 2017. Disponível em: https://www.embrapa.br/mandioca-e-fruticultura. Acesso em 10 jun.2017.

NEVES, M. F.; JANK, M. S. Perspectivas da cadeia produtiva da laranja no Brasil: a agenda 2015. Pensa Boletim Online, São Paulo, 2006.

NEVES, M. F. et al. O retrato da citricultura brasileira. Ribeirão Preto: CitrusBR, 2010. 